\title{
SPECTRUM OF THE PRODUCT OF OPERATORS
}

\author{
MILAN HLADNIK AND MATJAŽ OMLADIČ
}

(Communicated by John B. Conway)

\begin{abstract}
It is shown that the product of two operators on a Hilbert space has real spectrum if one of them is symmetric and the other is positive. Also, the product of two positive operators has positive spectrum.
\end{abstract}

In general we do not know much about the spectrum of the product of two (bounded, linear) operators. The Jacobson Lemma which states that the two products $A B$ and $B A$ of operators $A$ and $B$ have the same spectrum except possibly for the point zero, is one of the few known facts. In finite dimensions there are results under additional hypotheses that, say, operators $A$ and $B$ are orthogonal projections [1] (see also [3]) or, that they are strictly positive [2]. Here, we generalize a finite-dimensional result of Hu-yun [2] on the spectrum of the product of two positive operators.

Our first result, Proposition 1, is an immediate consequence of the following obvious facts: (1) If $B$ is a surjective operator on a Hilbert space $H$ such that the range of $B^{*}$ is dense in $H$, then $B$ is invertible. (2) If $A$ and $B$ are two operators on $H$ such that the product $B A$ is invertible and 0 is not in the residual spectrum of $B^{*}$, then $B$ is invertible. Here and in the sequel $A^{*}$ denotes the Hilbert space adjoint of the operator $A$. Besides, " 0 is in the residual spectrum of an operator $A$ " means that $A$ has trivial kernel and its range is not dense in $H$.

Proposition 1. Let $A$ and $B$ be operators on a Hilbert space $H$, let $B$ be positive and denote by $P$ the positive square root of the operator $B$. Then,

$$
\sigma(A B)=\sigma(B A)=\sigma(P A P) .
$$

ProOF. The nonzero points of the spectrum of the two products $A B$ and $B A$ coincide by the Jacobson Lemma. If $B A$ is invertible, use assertion (2) above to get that $A B$ is also invertible. To get the other implication, use the first one for the adjoints. The second equality stated in the proposition follows from the fact that $A B=(A P) P$ using the above argument for the product of $A P$ and $P$.

REMARK. Observe that it can easily be proved that in the case $\operatorname{Ker} A^{*}=$ $\operatorname{Ker} A=\operatorname{Ker} B=\{0\}$, the operators $A B$ and $P A P$ are quasisimilar in the sense of Sz.-Nagy and Foias [4]. However, even when $A$ and consequently $P A P$ are selfadjoint, the standard theory of quasisimilarity gives us only the inclusion $\sigma(P A P) \subset$ $\sigma(A B)$ in general.

In the following we shall need the notion of the numerical range $W(S)$ of an operator $S$ on $H$, defined as the set $\{(S x, x) ;\|x\|=1\}$ where $(\cdot, \cdot)$ denotes the

Received by the editors May 21, 1986 and, in revised form, October 22, 1986.

1980 Mathematics Subject Classification (1985 Revision). Primary 47A10.

This work was supported by the Research Council of Slovenia.

(C) 1988 American Mathematical Society $0002-9939 / 88 \$ 1.00+\$ .25$ per page 
inner product of the space $H$. It is well known that for a normal operator $S$ the closure of the set $W(S)$ is the closed convex hull of the spectrum $\sigma(S)$. If $S$ is selfadjoint, denote also $m(S)=\inf W(S)$ and $M(S)=\sup W(S)$.

Proposition 2. Let $A$ be a selfadjoint operator, let $B$ be a positive operator, and denote by $P$ the positive square root of $B$. Then

(a) if $m(A)<0<M(A)$, then $W(P A P)$ is contained in the interval $[M(B) m(A)$, $M(B) M(A)]$,

(b) if $A$ is positive, then $W(P A P)$ is contained in $[m(A) m(B), M(A) M(B)]$,

(c) if $A$ is negative, then $W(P A P)$ is contained in $[m(A) M(B), M(A) m(B)]$.

ProOF. The proof of case (a) will be given only. Since $m(A)<0<M(A)$, it holds for any vector $x \in H$ that

$$
\begin{aligned}
(P A P x, x) & =(A P x, P x) \leq M(A)\|P x\|^{2}=M(A)(P x, P x) \\
& =M(A)(B x, x) \leq M(A) M(B)\|x\|^{2}
\end{aligned}
$$

and also

$$
\begin{aligned}
(P A P x, x) & =(A P x, P x) \geq m(A)\|P x\|^{2}=m(A)(P x, P x) \\
& =m(A)(B x, x) \geq m(A) M(B)\|x\|^{2} .
\end{aligned}
$$

Both propositions together give us an interval in which the spectra of the products $A B$ and $B A$ of a selfadjoint operator $A$ and a positive operator $B$ are contained. The endpoints of this interval may or may not belong to the spectrum of the product $A B$, as it can be seen by taking appropriate multiplication operators on the space $L^{2}[0,1]$.

Recall now the following result of Hu-yun [2]. Denote by $\mu_{i}, \nu_{i}$, and $\lambda_{i}$ respectively the eigenvalues of the $n \times n$ matrices $A, B$, and $A B$ where $A$ and $B$ are strictly positive. Then,

$$
\frac{2}{n} \frac{\left(\min \mu_{i}\right)^{2}\left(\min \nu_{i}\right)^{2}}{\left(\min \mu_{i}\right)^{2}+\left(\min \nu_{i}\right)^{2}}<\lambda_{i}<\frac{n}{2}\left[\left(\max \mu_{i}\right)^{2}+\left(\max \nu_{i}\right)^{2}\right]
$$

Our Proposition 2(b) gives another estimate

$$
\left(\min \mu_{i}\right)\left(\min \nu_{i}\right) \leq \lambda_{i} \leq\left(\max \mu_{i}\right)\left(\max \nu_{i}\right)
$$

which is sharper even in the case $n=2$.

We have thus seen that the spectrum of the product of a selfadjoint and a positive operator is always real. A natural question arises whether the product of two selfadjoint operators has always real spectrum. The answer is no even in two dimensions. Namely, take

$$
A=\left[\begin{array}{cc}
1 & 0 \\
0 & -1
\end{array}\right], \quad B=\left[\begin{array}{ll}
0 & 1 \\
1 & 0
\end{array}\right]
$$

to get a product $A B$ with purely imaginary spectrum.

ACKNOWLEDGMENT. The authors are grateful to the referee for his valuable comments. 


\section{REFERENCES}

1. W. N. Anderson, Jr., E. J. Harner and G. E. Trapp, Eigenvalues of the difference and product of projections, Linear Multilinear Algebra 17 (1985), 295-299.

2. Sh. Hu-yun, Estimation of the eigenvalues of $A B$ for $A>0, B>0$, Linear Algebra Appl. 73 (1986), 147-150.

3. M. Omladic, Spectra of the difference and product of projections, Proc. Amer. Math. Soc. 99 (1987), 317-318.

4. B. Sz.-Nagy and C. Foias, Harmonic analysis of operators on Hilbert space, North-Holland, Amsterdam, 1970.

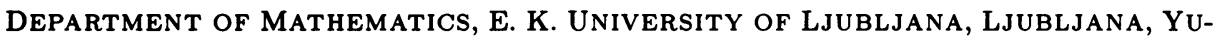
GOSLAVIA 